\title{
Fluid mobility and frequency-dependent seismic velocity - Direct measurements
}

\author{
Michael L. Batzle ${ }^{1}$, De-Hua Han², and Ronny Hofmann ${ }^{1}$
}

\begin{abstract}
The influence of fluid mobility on seismic velocity dispersion is directly observed in laboratory measurements from seismic to ultrasonic frequencies. A forceddeformation system is used in conjunction with pulse transmission to obtain elastic properties at seismic strain amplitude $\left(10^{-7}\right)$ from $5 \mathrm{~Hz}$ to $800 \mathrm{kHz}$. Varying fluid types and saturations document the influence of pore-fluids. The ratio of rock permeability to fluid viscosity defines mobility, which largely controls pore-fluid motion and pore pressure in a porous medium. High fluid mobility permits pore-pressure equilibrium either between pores or between heterogeneous regions, resulting in a low-frequency domain where Gassmann's equations are valid. In contrast, low fluid mobility can produce strong dispersion, even within the seismic band. Here, the low-frequency assumption fails. Since most rocks in the general sedimentary section have very low permeability and fluid mobility (shales, siltstones, tight limestones, etc.), most rocks are not in the lowfrequency domain, even at seismic frequencies. Only those rocks with high permeability (porous sands and carbonates) will remain in the low-frequency domain in the seismic or sonic band.
\end{abstract}

\section{INTRODUCTION}

Seismic and acoustic methods are among the primary tools used in oil exploration, reservoir delineation, and recovery processes monitoring. Numerous theories on wave propagation have been developed, but these concepts remain remarkably unencumbered by the measured data needed to prove, delimit, or extend them. Not only do these concepts need calibration, but the fundamental parameters controlling wave propagation also must be understood. For example, Biot inertial mechanisms (Biot, 1956) have been contrasted to local or squirt mechanisms by many authors (Mavko and Nur, 1975; O'Connell and Budiansky, 1977; Dvorkin and Nur, 1993; Dvorkin et al., 1995). Ursin and Toverud (2002) compare many of the proposed dispersion-attenuation relations but conclude it is difficult to discriminate among mechanisms over the narrow seismic band. Berryman and Wang (2000) and Pride and Berryman (2003) develop their own dual porosity model but have no data to test their dispersion prediction. Similarly, Chapman et al. (2002) produce a crack-andpore model that predicts potentially large compressional and shear dispersions but have no measured information to evaluate their concepts. In contrast, King and Marsden (2002) rely on poroelastic models for their estimates of dispersion from measured, but narrowband, ultrasonic data.

Laboratory measurements provide much of the information on basic rock properties. Compressional and shear velocities have been collected in laboratories for many years; but because of the ultrasonic or high-amplitude stress-strain methods in common use, the vast bulk of the data are far outside either the seismic or logging frequency and amplitude ranges. Considerable effort is expended reconciling velocity values observed with these different techniques. Even in a completely homogeneous rock, frequency-dependent velocities, or dispersion, yield inconsistent values between different measurement bands. This dispersion is a complex function of heterogeneity, pore-fluid properties, and mobility. On the other hand, with sufficient information, dispersion could itself be used as a fluid indicator or as a remote measurement of permeability.

Fluid mobility determines pore-pressure distribution as a fully saturated rock is deformed slightly when a seismic wave passes. Thus, seismic properties are influenced not only by the kind of pore fluid but also by its ability to move within the rock. We define fluid mobility $M$ as

$$
M=\frac{k}{\eta}
$$

\footnotetext{
Manuscript received by the Editor September 12, 2003; revised manuscript received April 19, 2005; published online January 6, 2006.

${ }^{1}$ Colorado School of Mines, Department of Geophysics, 1500 Illinois St., Golden, Colorado 80401. E-mail: mbatzle@mines.edu; rhofmann@ mines.edu.

${ }^{2}$ University of Houston, Department of Geosciences, 4800 Calhoun Rd., Houston, Texas 77204. E-mail: De-hua.han@mail.uh.edu. (C) 2006 Society of Exploration Geophysicists. All rights reserved.
} 
where $k$ is permeability and $\eta$ is viscosity. For any frequency, if mobility is sufficiently low, pore pressure remains out of equilibrium, and we are necessarily in the high-frequency regime. This means that since most rocks in the sedimentary column have very low intrinsic permeabilities (i.e., shales, siltstones, tight limestones), even seismic frequencies for most rocks will be in the high-frequency regime.

White (1975) develops a model for inhomogeneous distributions of gas within rocks. This concept has been further developed by others into a more general description of partial gas saturation. Gist (1994), for example, defines a geometric factor dependent on gas-zone sizes and separation and relates this directly to the frequency dependence of velocity. Pride et al. (2003) point out that this behavior could also result from general inhomogeneities within the rock structure. Variations in rock compliance lead to heterogeneous pore pressures, resulting in fluid motion. In these cases, frequency dependence is a function of diffusion length:

$$
f_{t}=\frac{D}{L^{2}},
$$

where $f_{t}$ is the transition frequency, $D$ is the diffusivity, and $L$ is the diffusion-length scale. The diffusivity can be estimated from

$$
D=\frac{K_{f} M}{\phi}
$$

where $K_{f}$ is the fluid bulk modulus and $\phi$ is porosity.

The measurements and applications of rock elastic and anelastic properties cover many orders of magnitude in both frequency and amplitude. Figure 1 shows the relative locations of many of the common techniques in frequency and amplitude space. Exploration seismic data are usually collected between 10 and $100 \mathrm{~Hz}$ and at strain amplitudes around $10^{-7}$. Sonic well logs are usually in the same amplitude range, but logging frequencies are often in the tens of kilohertz. Lowamplitude ultrasonic techniques easily applied in the laboratory, but these measurements are in the megahertz range. Any

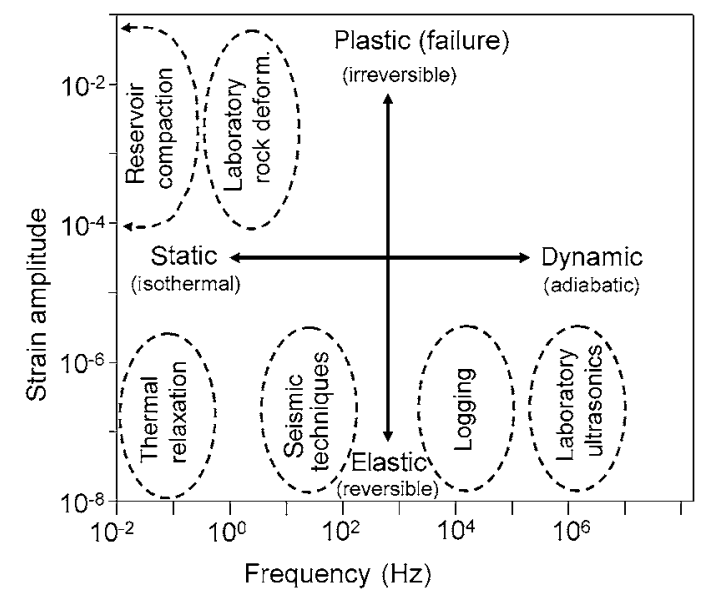

Figure 1. Generalized chart of deformation processes over a wide range of frequencies and amplitudes. Moduli or velocities measured in one amplitude/frequency domain are usually invalid in other domains since different deformation mechanisms are operating. significant velocity dispersion introduces systematic errors when comparing these different measurement techniques.

Significant discrepancies in velocity are often found when comparing sonic logs to checkshot or vertical seismic profile (VSP) surveys. De et al. (1994) find velocities 1\%-7\% higher in sonic logs. Schmitt (1999) observes a much larger dispersion in heavy oil sands, where sonic log velocities are up to $20 \%$ higher than from a VSP. We should expect the discrepancy to be much greater with ultrasonic data. For example, Sams et al. (1997) use a mixture of VSP, sonic logs, and ultrasonic core measurements from a single borehole to extract dispersion and attenuation. They also observe velocity differences of up to $20 \%$. However, the application of such different techniques with different resolutions and scales complicates direct comparison.

At the other extreme, seismic velocities could be estimated from stress-strain measurements made on rock samples in the laboratory at seismic frequencies. However, the typical applied strain levels are normally orders of magnitude larger than those of a seismic wave. Discrepancies are usually on the order of factors of two or three (Tutuncu et al., 1998). Figure 2 shows the results from Iwasaki et al. (1978), where the measured modulus varies by two orders of magnitude, depending on the strain amplitude. The same problem applies if we try to go in the opposite direction - estimating the macroscopic mechanical properties of a formation from sonic logs. Different deformation mechanisms are active under different amplitude ranges. Figure 2 demonstrates clearly that measurement amplitudes must be of the same order of magnitude as any application; otherwise, the results may be invalid.

Several techniques are available for measuring the lowfrequency, low-amplitude elastic and viscoelastic properties of rocks. Most of these techniques have been used for many years by material scientists examining the properties of metals and ceramics. There are two basic experimental methods to make low-frequency measurements: resonance and stressstrain techniques. Resonant bar methods utilize a cylindrical or parallelepiped sample, usually driven into or through a

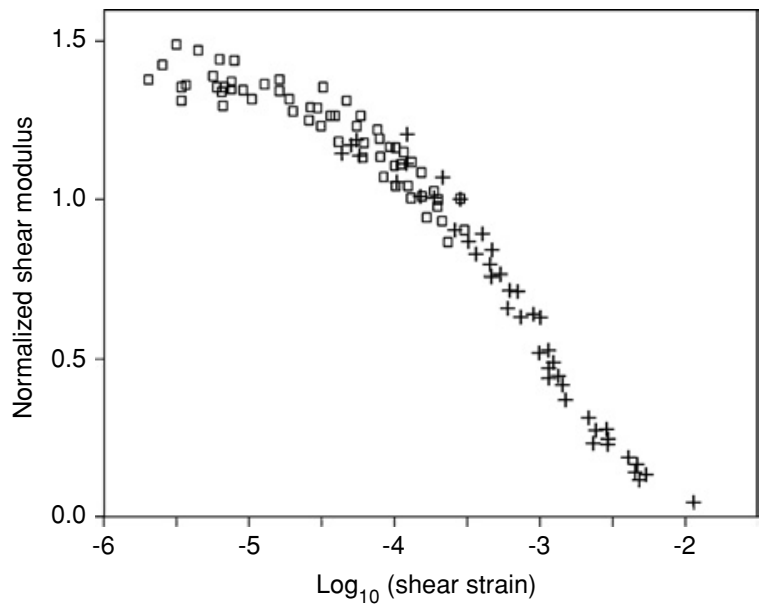

Figure 2. Measured shear modulus versus shear strain (after Iwasaki et al., 1978). Moduli are normalized to the value measured at $10^{-4}$ strain amplitude. Squares and crosses represent two different measurement techniques. 
resonant vibration with a sinusoidal force. Numerous modes of vibration are possible, including length deformation, sensitive to the Young's modulus and flexural and torsional deformations, sensitive to the shear modulus. The moduli are calculated from the frequency of the resonance, density, and dimensions of the sample. Attenuation is determined by the width of the resonance frequency peak or by the decay of the resonation once the driving force is turned off. These techniques have the advantage of being fairly simple and robust. Winkler et al. (1979), Clark (1980), Tittmann et al. (1980), Murphy (1982), and Bulau et al. (1983) have used the resonant bar method to collect some of the first and most important low-frequency data. Yin et al. (1992) and Cadoret et al. (1995) have collected data in the kilohertz range, characterizing the fluid distribution in rock and sand samples.

This concept is extended in resonance ultrasound spectroscopy (RUS) by using a broad frequency range to capture numerous resonance peaks (Demarest, 1969; Ulrich et al., 2002; Zadler, et al., 2004). Identification of the modes excited allows one to determine the suite of elastic constants in a rock. In fact, RUS permits the suite of moduli for anisotropic rocks to be ascertained on a single sample using a single frequency sweep.

These resonance methods, however, have disadvantages. The rock samples must be sufficiently durable and homogeneous such that long, narrow bars can be machined. Larger samples result in lower frequencies. Winkler (1979), for example, requires a parallelepiped about $5 \mathrm{~cm}^{2}$ in cross section and almost $1 \mathrm{~m}$ in length. Another technique involves clamping passive masses to the ends of samples to lower the frequency yet keeping the sample size small. The frequencies that can be measured with typical resonance bars are usually limited to the primary resonance and a few overtones. This narrow bandwidth makes it difficult to discriminate among attenuation mechanisms and to quantify dispersion. The extended frequencies of the RUS technique make it sensitive to sample jacketing and suspension procedures. In addition, the flexural and torsional modes suffer from inhomogeneous strain conditions. White (1986) shows that macroscopic fluid motion resulting from inhomogeneous strain or inappropriate boundary conditions can significantly contaminate the measurements.

\section{LOW-FREQUENCY MEASUREMENT TECHNIQUES}

Forced deformation is an alternative approach where the stress-strain behavior of the rock is recorded. Stress-strain measurements have been made on rock samples to determine their macroscopic mechanical properties for nearly a century. However, these common measurements are made with deformation amplitudes several orders of magnitude larger than seismic amplitudes. As a result, the measured modulus and attenuation values usually differ from seismic values by factors of three and more (Figure 2). To make measurements at low amplitudes requires extremely sensitive deformation or displacement sensors. These sensors usually consist of magnetic, optical, or capacitive transducers. The experiment often takes the form of applying a weak sinusoidal stress to the sample and monitoring the deformation with the transducers. The ratio of the stress to the strain gives the moduli, and the area of the hysteresis loop or the phase angle between the stress and strain gives the attenuation. Gladwin and Stacey (1974) and Jackson and Paterson (1987) use this method, driving the sample in a torsional mode to gather shear modulus and attenuation data. Peselnick et al. (1979), Spencer (1981), Dunn (1986, 1987), and Brunner et al. (2003) use axial deformation to determine Young's modulus and attenuation data. This method has the advantage that a very broad, continuous frequency band can be measured. In addition, smaller and more varied samples can be used.

Several problems make the stress-strain method a difficult procedure. The deformations in the sample, particularly the phase angle or hysteresis loop measurements, are very sensitive to slight misalignment of the sample and applied stress. Misalignment can easily distort signals. The transducers themselves also cause limiting physical constraints. Large, bulky transducers make it difficult to place the equipment in a pressure vessel and apply elevated confining and pore pressures. Often, only one direction of deformation can be monitored, so compressional and shear data cannot be collected on the same sample.

We utilize the stress-strain approach using axial deformation with resistive strain gauges bonded directly to the sample for transducers. A schematic of the equipment is shown in Figure 3. Our system is similar to that developed by Spencer (1981). The frequency band is very broad, from about 1 to nearly $2000 \mathrm{~Hz}$. The strain gauges are compact and permit the application of confining and pore pressure. The bonded

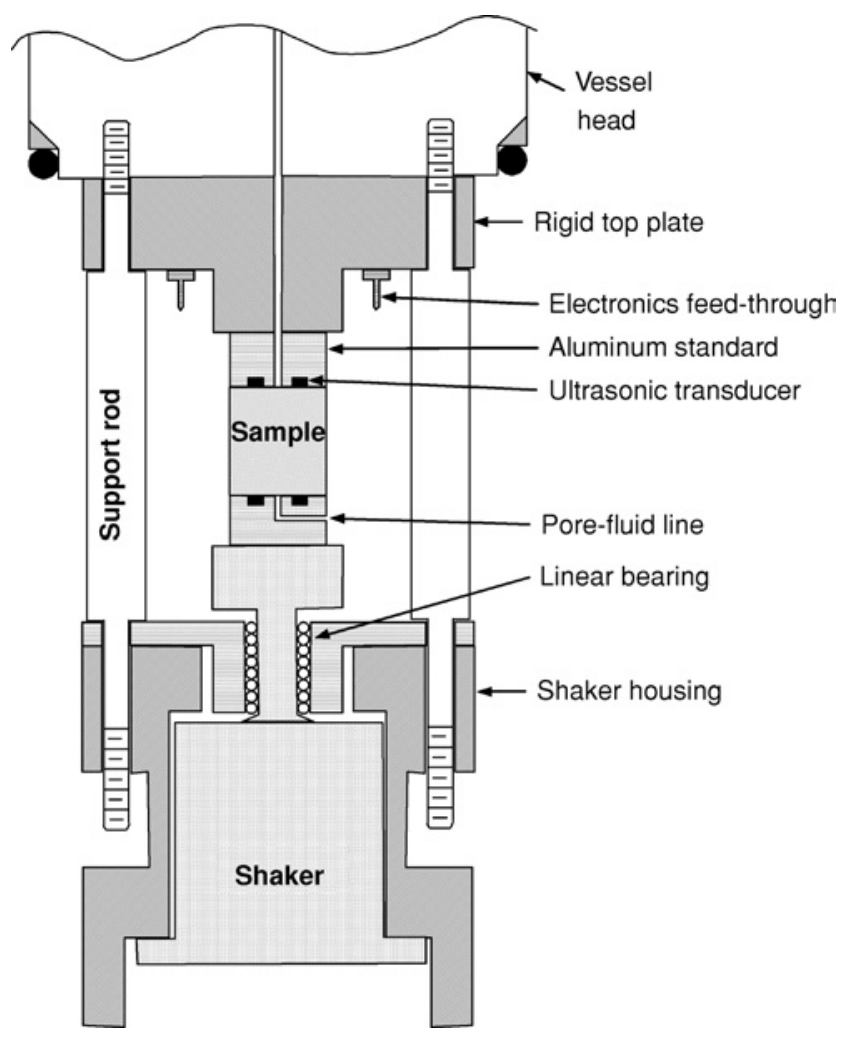

Figure 3. Schematic of the low-frequency measurement assembly. For seismic frequencies, strains are measured on both the sample and the aluminum standard. Ultrasonic transducers measure wave propagation near $800 \mathrm{kHz}$. Fluid lines permit control and exchange of pore fluids independent of confining pressure. 
gauges are less sensitive to equipment resonance than other transducers. One major advantage of these gauges is that they can be oriented on the sample to collect both axial and circumferential strain data (and other orientations). In addition, ultrasonic compressional and shear transducers are housed in the aluminum caps at the sample ends. As a result, both compressional and shear properties can be collected simultaneously over a frequency range of about $5 \mathrm{~Hz}$ to $800 \mathrm{kHz}$. The samples can be small; measurements can be made on common core plugs.

White (1986) points out that macroscopic fluid flow to the free boundary of the sample can also contribute significant error in these attenuation measurements. Dunn $(1986,1987)$ examines this flow in detail and confirms White's predictions. Because of the homogeneous strain and directly bonded pressure jacket in our apparatus, such macroscopic lateral fluid flow is prevented. Fluid movement within the small pore-fluid duct through the aluminum standards could affect the data. Such flow can be controlled by small valves in the pore-fluid lines next to the sample. On the other hand, this macroscopic flow can be important in distinguishing different dispersive mechanisms, the subject of a later paper. For the cases described here, the boundaries either remain closed or the sample permeabilities are so low that the boundary has no influence.

The major difficulties of this approach stem mostly from the very weak signals that must be processed, the sensitivity of the gauges to the surface preparation of the sample, and phase shifts resulting from misaligned loads in the sample column. The measured strains need to be on the order of $10^{-7}$ or less. To overcome this low signal level requires special shielding and high amplification with low-noise electronics (Figure 4). In addition, to measure the amplitude and phase of the applied stress, we use an aluminum standard in the sample column with similar strain gauges attached (Figure 3 ). Since aluminum is essentially elastic, its strain is exactly in phase with the stress. Both the aluminum standard and rock

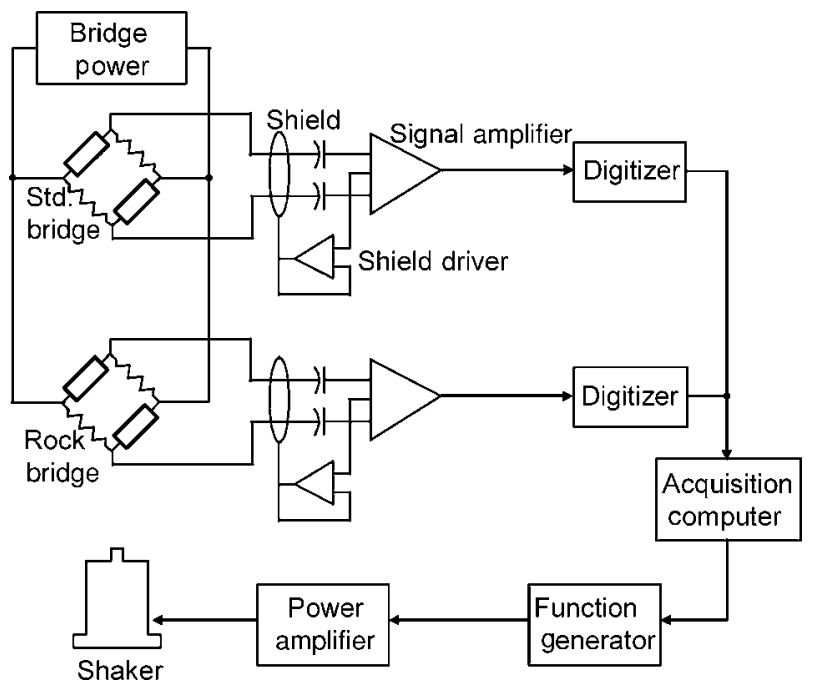

Figure 4. Schematic of the driving and acquisition circuit. Strain is measured using resistive gauges in Wheatstone bridges on the standard and sample. Feedback shield-driver amplifiers reduce noise at low strain amplitudes. sample signals go through identical electronic paths; amplifier phase shifts and other noise cancel in the analysis.

Typical acquired signals are shown in Figure 5 for both axially and circumferentially (vertically and horizontally) oriented gauges. Typically, several hundred cycles are digitized and averaged. The magnitude and phase angles are determined from the fast Fourier transforms of the signals. Although our equipment is capable of generating, recording, and analyzing stress-strain signals up to several tens of kilohertz, we are limited to a maximum of about $2500 \mathrm{~Hz}$ in the lowfrequency range because of resonances in the mechanical system. To measure at frequencies intermediate to our seismic and ultrasonic ranges, we would need to use resonant techniques on separate samples (Zadler et al., 2004). Surface problems (bad bonding, epoxy degradation, etc.) and column resonances usually result in obvious distortion of the sine waves. Stress misalignment is commonly indicated by inconsistent phase angles for different gauges on the sample or the standard. Under normal circumstances, we can measure Young's modulus to within about $5 \%$ and attenuation $\left(1 / Q_{E}\right)$ to within about 0.0018 .

\section{Errors and data inconsistencies}

Factors associated with the electronics, sensors, sample preparation, and samples themselves lead to errors or inconsistencies in the measured elastic properties. Most of the errors attributable to the electronics are eliminated because measurements are a comparison of parallel circuits (and circuits can be interchanged). However, the strain sensors themselves can contribute significant error. Although semiconductor strain gauges are very sensitive with a gauge factor of 155 , they can lack accuracy, varying by $5 \%$. Temperature and pressure sensitivities can be high. These variations in strain gauge response are mitigated somewhat by the use of similar gauges on the reference standard.

Sample preparation is another strong influence. Sealing the exterior surface of the sample is necessary to prevent porefluid flow across the boundary, to provide a bonding surface for the strain gauges, and to prevent diffusion of the nitrogen confining gas. Tests on known materials (see Experimental Procedure section) indicate that bonding and jacketing do not significantly affect the results.

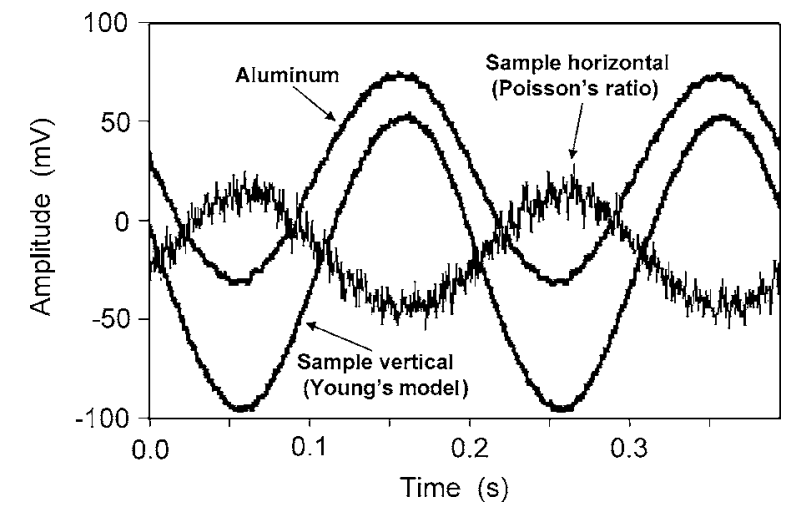

Figure 5. Measured strain bridge amplitudes at $5 \mathrm{~Hz}$ after several stages of amplification. The horizontal gauges require additional amplification because of the weaker signal - hence, the increased noise. 
Probably the largest source of inconsistency in our measurements is sample heterogeneity. All samples are heterogeneous (and anisotropic) to some degree. Ultrasonic wave propagation provides a bulk measurement of an entire sample's properties. On the other hand, low-frequency data are collected using strain gauges with dimensions of about $1 \times 6 \mathrm{~mm}$. These gauges may accurately measure the local moduli, but these measured moduli often differ from the bulk average. In extreme cases - for example, cemented streaks within a sample - average versus local properties can differ by factors of three or more. In our measurements, differences between ultrasonic and low-frequency results, particularly for the dry samples, are largely a result of sample heterogeneity.

\section{Experimental procedure}

The experimental procedure we use is an extensional stressstrain measurement. This is the classical Young's modulus. If the assumption of isotropic, homogeneous materials is valid, then we need collect only two independent elastic parameters. In our apparatus, we do not measure displacement (as do most other experimental setups) but only the strain on the sample surface. The axial strain $\varepsilon_{33}$ under our boundary conditions is given by

$$
\varepsilon_{33}=\frac{\partial u}{\partial x}=F \cos \left(\sqrt{\frac{\rho}{E}} \times \omega x\right) \sin (\omega t),
$$

where $u$ is displacement, $x$ is location, $\omega$ is frequency, $\rho$ is density, $E$ is Young's modulus, and $F$ is an elastic constant defined by the geometry. From the horizontal strain we can extract Poisson's ratio $v$, where the superscript $r x$ refers to the rock value:

$$
v^{r x}=-\frac{\varepsilon_{11}^{r x}}{\varepsilon_{33}^{r x}} .
$$

Axial strain measurements are also made on the aluminum standard (superscript al), and the rock's Young's modulus $E^{r x}$ is derived from the known Young's modulus of aluminum $E^{a l}$ and the ratio of the aluminum to rock strain:

$$
E^{r x}=E^{a l} \frac{\varepsilon_{33}^{a l}}{\varepsilon_{33}^{r x}} .
$$

Well-known relationships can be used to calculate the remaining elastic properties for an isotopic homogeneous sample:

$$
\begin{aligned}
\mu & =\frac{E}{2(1+v)}, \\
K & =\frac{E}{3(1-2 v)}, \\
V_{S} & =\sqrt{\frac{\mu}{\rho}} \\
V_{P} & =\sqrt{\frac{K+\frac{4}{3} \mu}{\rho}} .
\end{aligned}
$$

Here, $\mu$ and $K$ are the shear and bulk modului, and $V_{s}$ and $V_{p}$ are the shear and compressional velocities, respectively.

The consistency of our technique can be checked if we can measure strain over an extended frequency range on a simple, homogeneous material. For test purposes, we use an acrylic plastic or polymethylmethacrylate (Plexiglas). This material has a low modulus similar to rocks, yet it is homogeneous and easy to work with. The major drawback is that its composition can be quite variable from batch to batch; thus, the results cannot be directly compared to measurements on Plexiglas by other investigators. We made measurements with three techniques: stress-strain, resonant bar (on a separate sample), and ultrasonic wave propagation. The results can be seen in Figure 6. Thus for simple, isotropic, homogeneous material, all of these techniques give consistent results in velocity and attenuation.

In a linear viscoelastic medium, the velocity dispersion and attenuation are coupled. A schematic example is shown in Figure 7. For a single relaxation mechanism, such as pore fluids oscillating in a uniform system, compressional velocity (or bulk modulus) will show a sigmoidal increase with increasing frequency. Attenuation $(1 / Q p)$ will peak at the relaxation frequency, where velocity increases rapidly. This coupling can be described by a Cole-Cole (1941) or Kramers-Kronig relation Bourbie et al., 1987). In partially saturated rocks, the pore fluid is usually inhomogeneously distributed, sometimes referred to as patchy saturation. For such partial saturation (dotted line, Figure 7), velocity will increase when the frequency becomes too high for pressure equilibration to occur
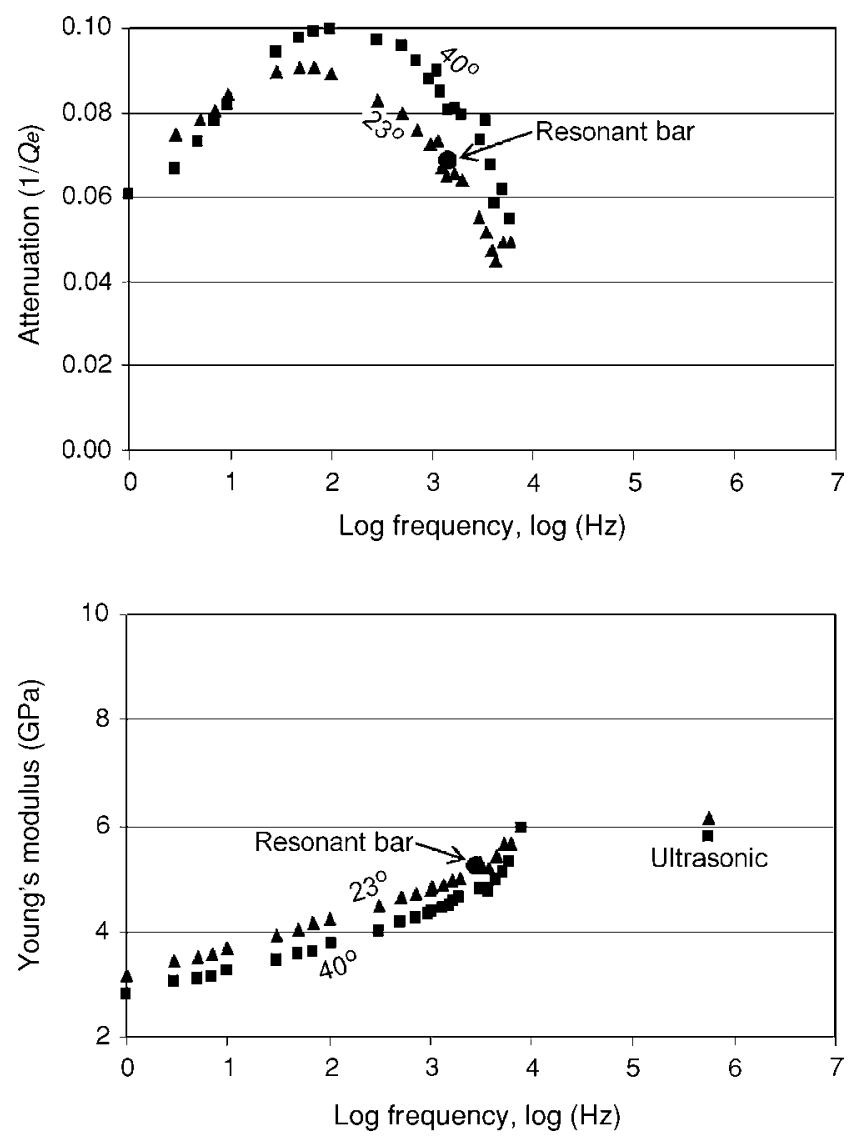

Figure 6. Measured Young's modulus and attenuation values for acrylic plastic (Plexiglas) at $23^{\circ}$ and $40^{\circ} \mathrm{C}$ using forced deformation, resonance, and ultrasonic techniques. For simple isotropic, homogeneous materials, the results are consistent. 
between fully saturated regions and regions of low saturation. Another mechanism, heterogeneous saturation on the scale of an ultrasonic wavelength, comes into play. Fully saturating the rock with liquid increases velocity. If the fluid mobility remains high, then the dispersion can occur in or above the seismic frequency band (dashed line, Figure 7). As mobility drops, the time required for pore fluids to come to pressure equilibrium increases, and the frequency band of the dispersion is lowered (solid line, Figure 7).

As an example of the velocity dispersion between high and low frequencies, the velocities measured on a North Sea sandstone as a function of brine saturation are shown in Figure 8. This sample has high porosity $(35 \%)$ and permeability $(8.7 \mathrm{D})$, but saturation is heterogeneous. Even at low saturations, ultrasonic frequencies show noticeably higher compressional velocity. At an increased saturation of about $80 \%$, ultrasonic velocities increase dramatically. However, the seismic velocities remain low and largely can be explained by Gassmann's (1951) equations. Only as the saturation approaches a few percent of full saturation does the low-frequency velocity increase to match those measured ultrasonically. We presume that at sonic logging frequencies an intermediate behavior will be observed (dashed line, Figure 8).

\section{Fluid mobility — permeability influence}

As we have seen, pore-fluid motion and pressure equilibration control velocity changes and seismic sensitivity to porefluid types. One obvious factor in equation 1 is permeability. Lower permeability should require longer times, or lower frequencies, for saturated rocks to relax.

In a single rock sample, the permeability can be altered substantially by slightly modifying the pore texture. Clays such as smectites are sensitive to pore-fluid salinity. Sample YM5154 has a high smectite content, and at high salinities the clay structure is collapsed (Figure 9b). At low salinities, water is absorbed and the clays expand (Figure 9a). Thus, we can

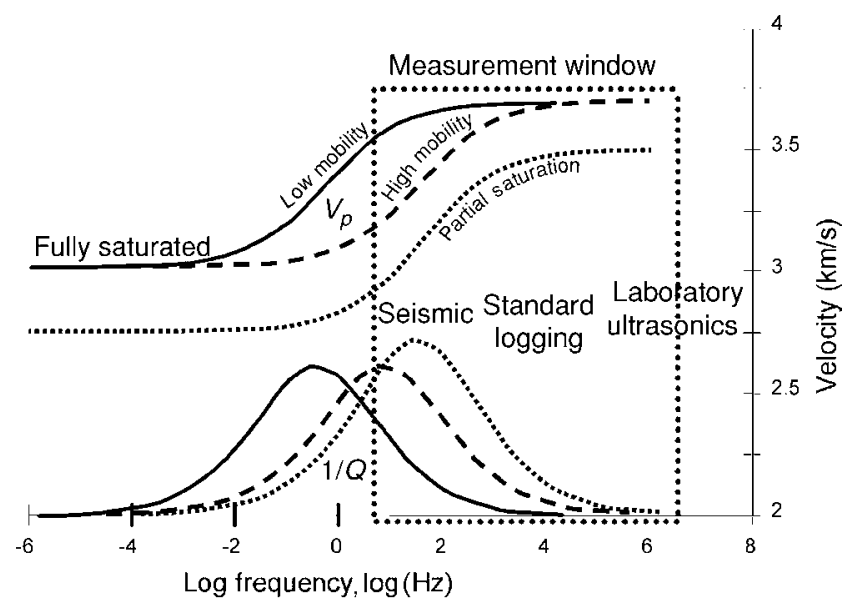

Figure 7. Schematic of the general frequency dependence of velocity $\left(V_{P}\right)$ and the associated attenuation $(1 / Q)$ for partially saturated rock and fully saturated rock with both low and high fluid mobility. Included in the box is the approximate frequency range of our laboratory measurements and how the frequency range corresponds to seismic and logging measurements. modify the pore space and the permeability by altering fluid salinity. Figure 10 shows the measured permeability with the schematic change in pore structure as salinity is modified. The process is largely reversible, and physical properties such as density, viscosity, and formation factor do not change significantly with clay expansion.

At high salinities and relatively high permeabilities, dispersion is strong and well within the seismic band (green points in Figure 11). The $V_{P}$ ranges from $2.5 \mathrm{~km} / \mathrm{s}$ at $5 \mathrm{~Hz}$ to $3.0 \mathrm{~km} / \mathrm{s}$ at $150 \mathrm{~Hz}$. Velocity continues to increase more slowly to $3.4 \mathrm{~km} / \mathrm{s}$ at ultrasonic frequencies. This dispersion occurs even at elevated pressures. Note that the seismic velocities are not within the low-frequency regime, nor do they agree with standard sonic values or those collected ultrasonically.

After freshwater is pushed through the sample, the clays swell and permeability drops by more than two orders of magnitude. Under these conditions, velocity dependence on frequency changes dramatically. As can be seen in Figure 11, ultrasonic velocities are little affected, and $V_{P}$ remains almost constant down through the seismic band. As far as this lowpermeability sample is concerned, seismic frequencies are ultrasonic frequencies.

\section{Fluid mobility — viscosity influence}

Fluid viscosity also strongly influences fluid motion and therefore fluid pressure and seismic velocity; it is the other obvious factor to test in equation 1 . The two most commonly used theoretical concepts to tie velocity to viscosity are the inertial coupling of Biot (1956) and the compliant pore coupling or squirt flow mechanism (see, e.g., O'Connell and Budiansky, 1977; Jones, 1986; Dvorkin and Nur, 1993; or Berryman and Wang, 2000). Biot gives a characteristic frequency for the fast compressional wave $\omega_{c}$ (roughly the boundary between

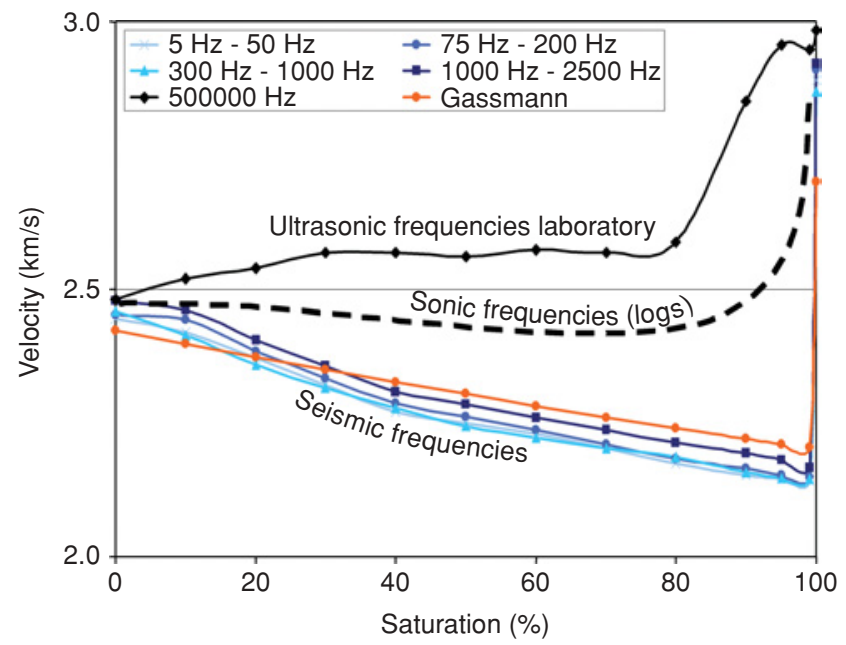

Figure 8. Measured and estimated velocities in sandstone as a function of water saturation by injection. Seismic frequency (blue) and ultrasonic (solid black) data are measured. Estimates of the velocities using Gassmann's equation and the initial dry moduli are shown in orange. The estimated values for a standard sonic log are shown as the dashed black line. 
a)

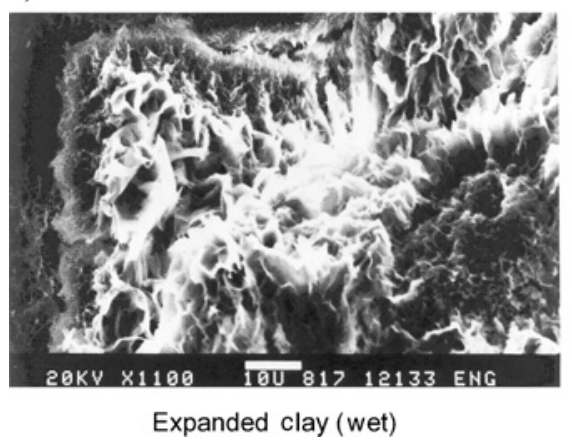

b)

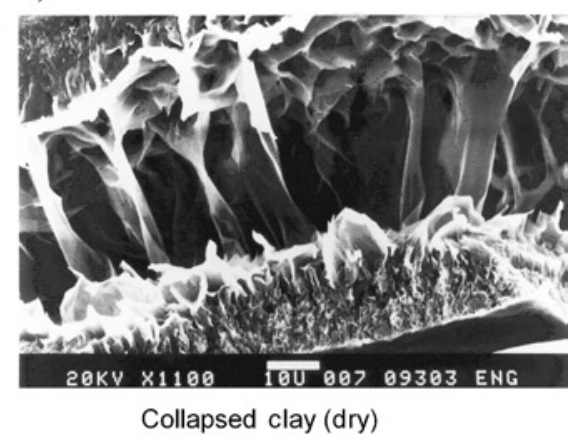

Figure 9. Scanning electron microscope images of the pore space and clay fabric of sample YM5154. The clay fabric as received was collapsed as a result of clays losing water (right). This more open fabric persists for high salt-concentrated brines filling the pores. When distilled water is injected, the clays swell blocking the pores. Scale is indicated by the white $10-\mu \mathrm{m}$ bar just below the image.

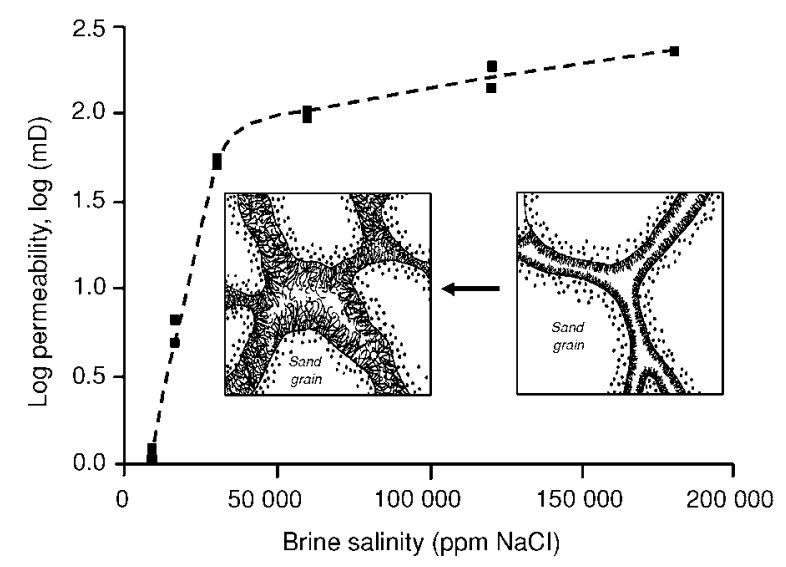

Figure 10. Measured permeability as a function of pore-fluid salinity. At about 50000 ppm sodium chloride concentration, the clays begin to swell and dramatically lower the permeability. The inset figures (after Neasham, 1977) show schematically the change in clay fabric.

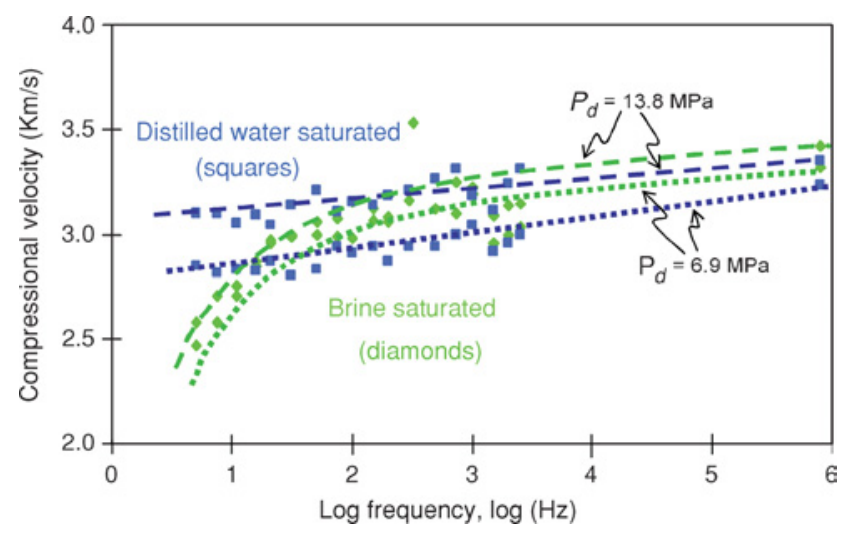

Figure 11. Measured compressional velocity as a function of frequency at two pore-fluid salinities and two differential pressures $\left(P_{d}\right)$. At high salinity, the permeability remains relatively high; significant dispersion is observed within the seismic band. At low salinity (distilled water), the low permeability and low fluid mobility force the dispersive region to lower frequencies (below our measurement window). high and low range) with the viscosity dependence $\eta$ in the numerator:

$$
\omega_{c}=\frac{\eta \phi}{k \rho} .
$$

Here, $\phi$ is porosity, $k$ is permeability, and $\rho$ is fluid density. However, squirt-flow mechanisms lead to viscosity dependence in the denominator (O'Connell and Budiansky, 1977):

$$
\omega_{c}=\frac{K \alpha^{3}}{\eta},
$$

where $K$ is frame modulus and $\alpha$ is crack aspect ratio (permeability is not an explicit term in this squirt critical frequency). These contrasting dependencies indicate viscosity can help ascertain which theory is applicable.

Figure 12 shows the strong dependence on temperature of glycerine viscosity. Other properties, such as bulk modulus, change as well but not by same the order of magnitude as viscosity.

Compressional $\left(V_{P}\right)$ and shear $\left(V_{S}\right)$ velocities for a sample of the Upper Fox Hills Sandstone (Heather well) are shown in Figure 13. Several features should be noted. For the dry sample (open symbols), $V_{P}$ and $V_{S}$ show little frequency or temperature influence. This confirms that the primary dispersive and temperature effects are dependent on pore fluids. When saturated with glycerine, strong temperature and frequency dependences are obvious. Shear velocity is not independent of the fluid but increases with increasing fluid viscosity, indicating a viscous contribution to the shear modulus. Also, $V_{P}$ increases with viscosity. More importantly, the dispersion curve shows a systematic shift to lower frequencies with increasing viscosities, consistent with squirt flow. Vo-Thant (1990) sees a similar dramatic shear velocity decrease with temperature in glycerol-saturated rocks but at restricted frequencies in the kilohertz range.

An example of a rock saturated with a natural highly viscous pore fluid is shown in Figure 14. This porous carbonate $(\phi=0.24, k=550 \mathrm{mD})$ contains a very heavy oil $(\rho=$

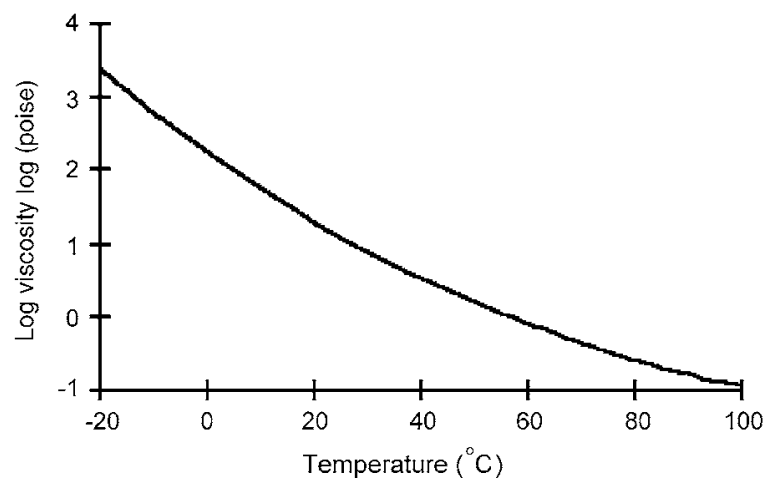

Figure 12. Glycerine viscosity as a function of temperature (after Gallant et al., 1984). Although properties such as modulus and density increase with decreasing temperature, their changes are small compared to the orders of magnitude of variation in viscosity. 
1.12, API $=-5)$. At low temperature $\left(25^{\circ} \mathrm{C}\right)$, the hydrocarbon is almost solid. Compressional and shear velocities are high, and there is little dispersion. At higher temperatures, viscosity drops and dispersion becomes substantial, even within the seismic frequency band. The increase in velocity between the seismic and ultrasonic bands is about $50 \%$ for both $V_{P}$ and $V_{S}$. This is consistent with the $20 \%$ change between VSP and sonic-log velocity seen in similar material by Schmitt (1999). The high viscosity and thus low fluid mobility at low temperature places this rock-fluid combination in the high-frequency domain, even for seismic frequencies (Figure 7). Thus,

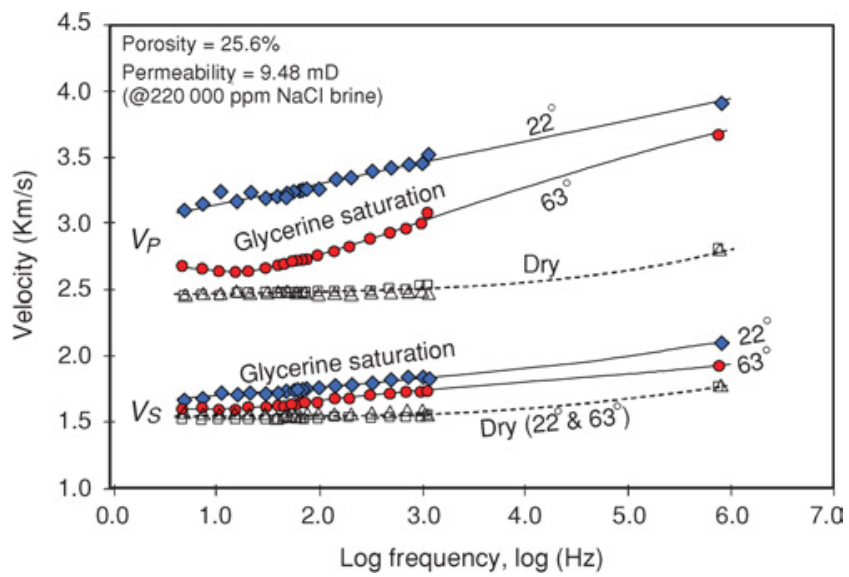

Figure 13. Compressional and shear velocities as a function of frequency and temperature for dry and glycerine-saturated Foxhills Sandstone. For the dry sample (open symbols), there is little temperature and frequency dependence. The difference between the dry ultrasonic and low-frequency values is probably the result of sample heterogeneity. Upon saturation with glycerine, dispersion becomes apparent. Low-viscosity glycerine $\left(63^{\circ} \mathrm{C}\right)$ has little dispersion within the seismic band, but it is significant between seismic, logging, and ultrasonic bands. At high viscosity $\left(22^{\circ} \mathrm{C}\right)$, dispersion is evident within the seismic band. Note that shear velocity (and shear modulus) increases with saturation.

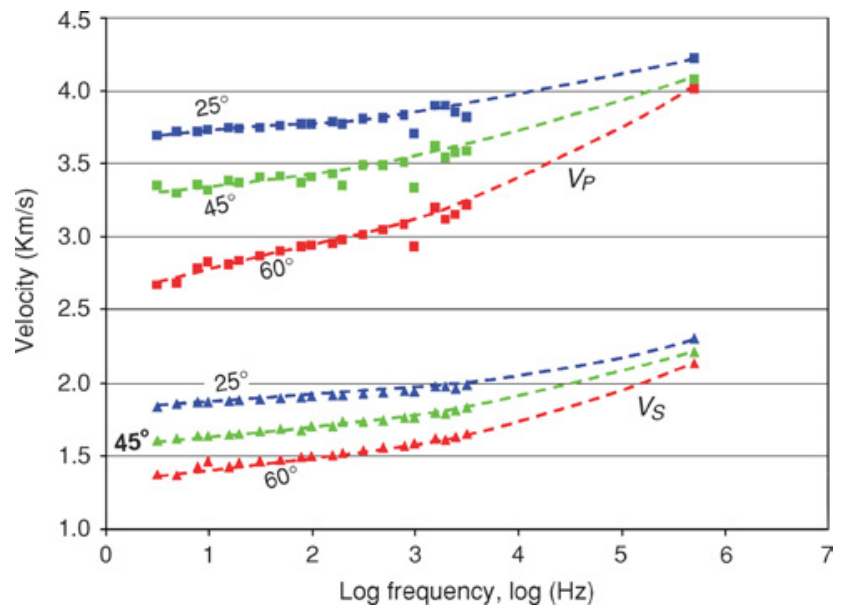

Figure 14. Compressional and shear velocities as a function of frequency and temperature for heavy oil-saturated Uvalde Carbonate. Velocity decreases with increasing temperature. Dispersion becomes substantial at $60^{\circ} \mathrm{C}$, even within the seismic band.

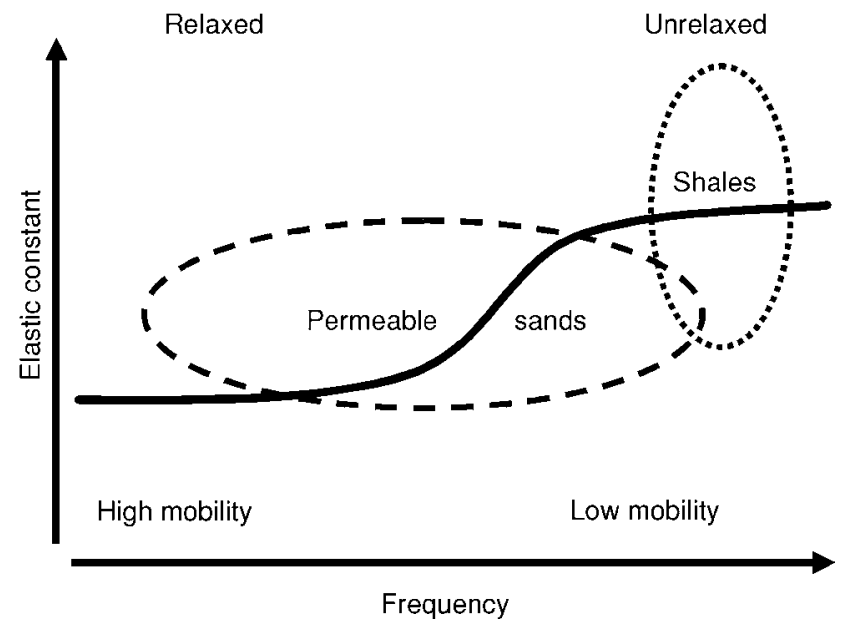

Figure 15. Schematic relation among elastic moduli (or velocity) and frequency and fluid mobility. At low mobility, pore pressure remains unrelaxed, even at seismic frequencies. Hence, for low-permeability shales, even seismic frequencies are unrelaxed or ultrasonic. Only the more permeable rocks can be unrelaxed and remain in the low-frequency domain.

viscosity must be included in fluid properties to model such rock behavior properly (Eastwood, 1993).

\section{DISCUSSION AND CONCLUSIONS}

These measurements over a broad frequency band demonstrate that velocity dispersion can be significant and is strongly influenced by fluid mobility. Mesoscopic fluid motion between heterogeneous compliant regions is one possible controlling process. Mechanisms coupling velocity to local pore-fluid motion driven by compliant pores (sometimes referred to as squirt flow) are also consistent with the data. The observed dependence on viscosity indicates that the inertial coupling mechanism for the Biot fast wave is not the dominant mechanism controlling dispersion. Nor will this mechanism control the associated attenuation in fully saturated rocks. This means that using the fast-wave crossover frequency to ascertain if a particular measurement is in the high- or low-frequency domain is incorrect. Also of importance is the observed increase in shear velocity after saturation with a high-viscosity fluid. This requires an increase in shear modulus with saturation, which violates fundamental aspects of Gassmann's equation. One missing component in many of these relations is the viscous skin depth of the shear wave. At high viscosity, fluid viscosity begins acting like a shear modulus. Particularly over the short distances within a pore space, viscous fluids will effectively support a shear wave.

The generalized frequency dependence on mobility indicated by our results is shown in Figure 15. This plot further generalizes Figure 7 and shows velocities as a function of fluid mobility as well as frequency. Lowering mobility increases the relaxation time needed for fluid equilibration, thus lowering the dispersion frequency. Since fluid permeability and viscosity can span several orders of magnitude, mobility also can vary by numerous orders of magnitude. Most sedimentary rocks - shales, siltstones, tight sandstones and carbonates, heavy oil sands, and evaporates (Shales in Figure 15) - have very low permeability and thus low fluid mobility. Thus, most 
rocks fall in the high-frequency regime, even in typical seismic exploration frequencies. This implies that for these rocks, seismic, sonic logging, and ultrasonic measurements may yield consistent velocity values (excluding issues with heterogeneity). This is even the case for permeable rock saturated with viscous oil. In contrast, porous and permeable sands and carbonates can fall anywhere on the dispersion curve (Permeable Sands in Figure 15). These include many of the most interesting reservoir rocks. As we have seen, there can be strong discrepancies among the different measurement techniques.

\section{ACKNOWLEDGMENTS}

This work was sponsored primarily through the Geophysical Properties of Fluids Consortium at the Colorado School of Mines. Billy J. Smith designed and built much of the lowfrequency device. John Castagna participated in several of the related research projects. Kurt Nihei and Jim Berryman provided useful comments and corrections to the original manuscript. Steve Pride was particularly helpful in his assessment of the results and discussion of potential relaxation mechanisms.

\section{REFERENCES}

Berryman, J. G., 1999, Origin of Gassmann's equation: Geophysics, 64, 1627-1629.

Berryman, J. G., and H. F. Wang, 2000, Elastic wave propagation and attenuation in a double-porosity, dual-permeability medium: International Journal of Rock Mechanics and Mineral Science, 37, 6378.

Biot, M. A., 1956, Theory of propagation of elastic waves in a fluidsaturated porous solid: II - Higher frequency range: Journal of the Acoustic Society of America, 28, 179-191.

Brunner, W. M., I. C. Getting, and H. A. Spezler, 2003, Device for the independent verification of subresonant mechanical damping measurements: Review of Scientific Instrumentation, 74, 1-7.

Bourbie, T., O. Coussy, and B. Zinszner, 1987, Acoustics of porous media: Gulf Publishing.

Bulau, J. R., S. R. Tittmann, and M. Abdel-Gawad, 1983, Attenuation and modulus dispersion in fluid saturated sandstone (abstract): EOS, Transactions of the American Geophysical Union, 64, 323324.

Cadoret, T., D. Marion, and B. Zinszner, 1995, Influence of frequency and fluid distribution on elastic wave velocities in partially saturated limestones: Journal of Geophysical Research, 100, 9789-9803.

Chapman, M., S. V. Zatsepin, and S. Crampin, 2002, Derivation of a microstructural poroelastic model: Geophysical Journal International, 151, 427-451.

Clark, V. A., 1980, Effects of volatiles on seismic attenuation and velocity in sedimentary rocks: Ph.D. dissertation, Texas A\&M University.

Cole, K. S., and R. H. Cole, 1941, Dispersion and absorption in dielectrics: Journal of Chemical Physics, 9, 341-351.

De, D. S., D. F. Winterstein, and M. A. Meadows, 1994, Comparison of P- and S-wave velocities and Q's from VSP and sonic log data: Geophysics, 59, 1512-1529.

Demarest, H. Jr., 1969, Cube-resonance method to determine the elastic constants of solids: Journal of the Acoustic Society of America, 49, 768-775.

Dunn, K.-J., 1986, Acoustic attenuation in fluid-saturated porous cylinders at low frequencies: Journal of the Acoustic Society of America, 79, 1709-1721.

- 1987, Sample boundary effect in acoustic attenuation of fluidsaturated porous cylinders: Journal of the Acoustic Society of America, 81, 1259-1266.

Dvorkin, J., and A. Nur, 1993, Dynamic poroelasticity: a unified model with the squirt and the Biot mechanisms: Geophysics, 58, 524533.

Dvorkin, J., G. Mavko, and A. Nur, 1995, Squirt flow in fully saturated rocks: Geophysics, $\mathbf{6 0}, 97-107$.

Eastwood, J., 1993, Temperature-dependent propagation of P- and S-waves in Cold Lake oil sands: Comparison of theory and experiments: Geophysics, 58, 863-872.
Gallant, R. W., C. L. Yaws, and X. Pan, 1984, Propylene glycols and glycerine, in R. W. Gallant and C. L. Yaws, eds., Physical properties of hydrocarbons, 2nd ed.: Gulf Publishing Company.

Gassmann, F., 1951, Uber die elastizität poroser medien: Der Natur Gesellschaft, 96, 1-23.

Gist, G. A., 1994, Interpreting laboratory velocity measurements in partially gas-saturated rocks: Geophysics 59, 1100-1109.

Gladwin, M. T., and F. D. Stacey, 1974, Anelastic degradation of acoustic pulses in rock: Physical Earth and Planetary Interiors, 8, 332-336.

Iwasaki, T., F. Tatsuoka, and Y. Takagi, 1978, Shear moduli of sand under cyclic torsional shear loading: Soils and Foundations, 18, 3956.

Jackson, I., and M. S. Paterson, 1987, Shear modulus and internal friction of calcite rocks at seismic frequencies: Pressure, frequency, and grain size dependence: Physical Earth and Planetary Interiors, 45, 349-367.

Jones, T., 1986, Pore fluids and frequency-dependent wave propagation in rocks: Geophysics, 56, 1939-1953.

King, M. S., and R. J. Marsden, 2002, Velocity dispersion between ultrasonic and seismic frequencies in brine-saturated reservoir sandstones: Geophysics, 67, 254-258.

Mavko, G., and A. Nur, 1975, Melt squirt in the asthenosphere: Journal of Geophysical Research, 80, 1444-1448.

Murphy, W. F., 1982, Effects of microstructure and pore fluids on the acoustic properties of granular sedimentary materials: Ph.D. dissertation, Stanford University.

Neasham, N. J., 1977, The morphology of dispersed clay in sandstone reservoirs and its effect on sandstone shaliness, pore space, and fluid flow properties: SPE paper 6858.

O'Connell, R. J., and B. Budiansky, 1977, Viscoelastic properties of fluid-saturated cracked solids: Journal of Geophysical Research, 82, 5719-5735.

Peselnick, L., H.-P. Liu, and K. R. Harper, 1979, Observations of details of hysteresis loops in Westerly Granite: Geophysical Research Letters, 6, 693-696.

Pride, S. R., and J. G. Berryman, 2003, Linear dynamics of doubleporosity dual-permeability materials: I - Governing equations and acoustic attenuation: Physics Review E, 68, 1-10.

Pride, S. R., J. M. Harris, D. L. Johnson, A. Mateeva, K. T. Nihei, R. L. Nowack, J. W. Rector, H. Spetzler, R. Wu, T. Yamamoto, et al., 2003, Permeability dependence of seismic amplitudes: The Leading Edge, 22, 518-525.

Sams, M. S., J. P. Neep, M. H. Worthington, and M. S. King, 1997, The measurement of velocity dispersion and frequency-dependent intrinsic attenuation in sedimentary rocks: Geophysics, 62, 14561464.

Schmitt, D. R., 1999, Seismic attributes for monitoring of a shallow heated heavy oil reservoir: A case study: Geophysics, 64, 368-377.

Spencer, J. W., 1981, Stress relaxation at low frequencies in fluid saturated rocks: Attenuation and modulus dispersion: Journal of Geophysical Research, 86, 1803-1812.

Tittmann, B. R., V. A. Clark, J. M. Richardson, and T. W. Spencer, 1980, Possible mechanisms for seismic attenuation in rocks containing small amounts of volatiles: Journal of Geophysical Research, 85, 5199-5208.

Tutuncu, A. T., A. L. Podio, A. R. Gregory, and M. M. Sharma, 1998, Nonlinear viscoelastic behavior of sedimentary rocks - Part I: Effect of frequency and strain amplitude: Geophysics, 63, 184-194.

Ulrich, T. J., K. R. McCall, and R. A. Guyer, 2002, Determination of elastic moduli of rock samples using resonant ultrasound spectroscopy: Journal of the Acoustic Society of America, 111, 16671674.

Ursin, B., and T. Toverud, 2002, Comparison of seismic dispersion and attenuation models: Studia Geophysica et Geodetica, 46, 293-320.

Vo-Thant, D., 1990, Effect of fluid viscosity on shear-wave attenuation in saturated sandstones: Geophysics, 55, 712-722.

White, J. E., 1975, Computed seismic speeds and attenuation in rocks with partial gas saturation: Geophysics, 40, 224-232.

, 1986, Biot-Gardner theory of extensional waves in porous rods: Geophysics, 51, 742-745.

Winkler, K., 1979, The effects of pore fluids and frictional sliding on seismic attenuation: Ph.D. dissertation, Stanford University.

Winkler, K. W., A. Nur, and M. Gladwin, 1979, Frictional sliding and seismic attenuation in rocks: Nature, 227, 528-531.

Yin, C. S., M. L. Batzle, and B. J. Smith, 1992, Effects of partial liquid/gas saturation on extensional wave attenuation in Berea Sandstone: Geophysical Research Letters, 19, 1399-1402.

Zadler, B. J., J. H. L. Le Rousseau, J. A. Scales, and M. L. Smith, 2004, Resonant ultrasound spectroscopy: Theory and application: Geophysical Journal International, 156, no., 154-169. 